\title{
MONOTONICITY OF WEIGHTED AVERAGES OF CONVEX FUNCTIONS
}

\author{
G. J. O. JAMESON
}

Abstract. We consider weighted averages of the form $B_{n}(W, f)=\sum_{r=0}^{n} w_{n, r} f(r / n)$, where $W$ is a summability matrix and $f$ is convex. Conditions are given for $B_{n}(W, f)$ to increase or decrease with $n$. It decreases whenever $W$ is a Hausdorff mean. The sequence of Bernstein polynomials for a convex function is a special case.

Mathematics subject classification (2010): 26D15, 40G05, 41A10.

Keywords and phrases: Monotonicity, weighted average, convex, summability matrix, Hausdorff mean, Bernstein polynomial.

\section{REFERENCES}

[1] S. Abramovich, G. Jameson and G. Sinnamon, Inequalities for averages of convex and superquadratic functions, J. Ineq. Pure Appl. Math., 5 (2004), issue 4, article 91 (electronic).

[2] G. BennetT, Inequalities complimentary to Hardy, Quart. J. Math. 49 (1998), 395-432.

[3] G. BENNETt, An inequality for Hausdorff means, Houston J. Math. 25 (1999), 709-744.

[4] G. BennetT, Mercer's inequality and totally monotonic sequences, Math. Ineq. Appl. 14 (2011), $747-775$.

[5] G. Bennett, Hausdorff means and moment sequences, Positivity 15 (2011), 17-48.

[6] G. Bennett And G. Jameson, Monotonic averages of convex functions, J. Math. Anal. Appl. 252 (2000), 410-430.

[7] J. Bustamante, Bernstein Operators and Their Properties, Birkhäuser (2017).

[8] R. A. De Vore And G. G. Lorentz, Constructive Approximation, Springer (1993).

[9] G. H. HaRdy, Divergent Series, Oxford Univ. Press (1949).

[10] I. J. Schoenberg, On variation diminishing approximation methods, On Numerical Approximation, Univ. Wisconsin Press (1959), 249-274. 